\title{
Combination of ENaC and CFTR mutations may predispose to cystic fibrosis-like disease
}

\section{To the Editors:}

The epithelial sodium channel $(\mathrm{ENaC})$ is part of the complex protein cystic fibrosis transmembrane conductance regulator (CFTR)-interactome that plays a key role in the composition of the airway surface liquid and the mucociliary clearance in the airways. We have recently screened $E N a C$ beta and gamma genes in 55 patients with diffuse bronchiectasis and with only one or no CFTR mutation/variant and showed that eight (15\%) patients carried at least one missense mutation in these $E N a C$ genes [1]. AzAD et al. [2] have also investigated the frequency of $\mathrm{ENaC}$ mutations in $\mathrm{ENaC}$ alpha, beta and gamma genes in a cohort of 76 patients with cystic fibrosis (CF)-like disease and with only one or no mutation in the CFTR gene. They found a significant increase in missense mutations or variants in patients (15.3\%) compared with controls (8.9\%) [2]. Moreover, a variant in $\mathrm{ENaC}$ alpha gene called p.W493R was found at a more than two-fold significantly increased incidence in patients $(8 \%)$ compared with controls (3\%) and, in the Xenopus laevis oocyte expression system, p.W493R was found to result in a more than four-fold higher $\mathrm{ENaC}$ activity.

We recently completed our previous study by screening exons and flanking intronic sequences of $E N a C$ alpha gene in the previously studied 55 patients (13 males and 42 females) with diffuse idiopathic bronchiectasis and with only one or no CFTR mutation/variant. We found three different intronic sequence variations (c.1242+54C $>$ T, c. $1553+32 \mathrm{G}>\mathrm{A}$ and c.1554-6C $>\mathrm{T}$ ) that are believed to be not deleterious and two different amino acid changes in heterozygous state in three patients of Caucasian origin: p.V462I in one patient and the p.W493R mutation extensively studied by AzAD et al. [2] in two patients. One of those also carried one CFTR variant (IVS8-5T) and one mutation in $E N a C$ beta gene (p.S82C). The main results from these three patients are summarised in table 1 .

It has been shown that the common human ENaC alpha polymorphism haA663T is a functional polymorphism that affects human ENaC surface expression, with lower channel activity and lower apparent number of active channels [3, 4]. Therefore, we studied the distribution of the haA663T genotypes in our group of 55 patients with diffuse bronchiectasis. We did not observe a significant increase in the presence of the haA663T genotype in our group (A663 allele; $69 \%$ ) compared with the expected frequency in controls (58$70.8 \%)[2,5]$.

To summarise the results from our 55 patients with bronchiectasis, $10(18 \%)$ patients carried at least one missense mutation in the $E N a C$ alpha (as outlined in this report), beta or gamma genes [1]. Among them, six had evidence of abnormal sodium transport, either in the sweat glands (sweat chloride concentration $40-59 \mathrm{mmol} \cdot \mathrm{L}^{-1}$ ) or in the nasal epithelium (basal nasal potential difference (PD) magnitude $>30 \mathrm{mV}$ or $<10 \mathrm{mV}$ ). Interestingly, three patients were transheterozygotes for CFTR/ $\mathrm{ENaC}$ mutations or variants: they carried either the p.F508del mutation or the IVS8-5T variant of the CFTR gene and all carried the p.S82C mutation in $E N a C$ beta gene; one of them also carried the p.W493R mutation in ENaC alpha gene (table 1). The basal PD in this patient was normal $(-13 \mathrm{mV})$ and no pharmacological study was performed. However, her sweat test was intermediate (44 mmol $\cdot \mathrm{L}^{-1}$ ) showing that the combination of the CFTR variant and the $E N a C$ mutations led to functional abnormalities.

Given the CF carrier incidence of $\sim 3.3 \%$ (one out of 30 ) and the 5T carrier incidence of $\sim 10 \%$, and the fact that $\sim 9 \%$ of the individuals in the general population is expected to carry a rare $\mathrm{ENaC}$ mutation, about one in $334(0.30 \%)$ of individuals are expected to be heterozygous for both a CF-causing CFTR mutation/variant and $E N a C$ missense mutation [2, 6, 7]. In 55 patients with diffuse bronchiectasis of unknown origin, we found an unexpected high proportion (5\%) of heterozygosity for both a CF-causing CFTR mutation/variant and ENaC mutation. MUTESA et al. [8] recently showed that, among five Rwandan patients with CF-like symptoms, elevated sweat chloride and with only one CFTR mutation identified, two of them carried one missense mutation in the $E N a C$ genes. Taken together, these results suggest that transheterozygosity for ENaC/CFTR mutations or variants may lead to deficient $\mathrm{ENaC} / \mathrm{CFTR}$ interaction, and abnormal ion transport in the airways and bronchiectasis.

\begin{tabular}{|c|c|c|c|c|c|c|c|}
\hline TABLE 1 & $\begin{array}{l}\text { Main } \mathrm{cl} \\
\text { alpha } \subseteq\end{array}$ & eristics of $t$ & ree patients & th idiopathic $b$ & רchiectasis bearin & a missense mut & in $\mathrm{ENaC}$ \\
\hline Age yrs & Sex & BMI $\mathbf{k g} \cdot \mathbf{m}^{-2}$ & FEV1 \% pred & CFTR mutation & $E N a C$ alpha mutation & $\mathrm{Cl}^{-}$sweat $\mathrm{mmol} \cdot \mathrm{L}^{-1}$ & Basal PD mV \\
\hline 62 & Female & 19 & 70 & None & p.V462l & 23 & -8 \\
\hline 78 & Female & 26 & 91 & None & p.W493R & 38 & -26 \\
\hline 66 & Female & 22 & 77 & IVS8-5T & p.W493R & 44 & -13 \\
\hline
\end{tabular}

BMI: body mass index; FEV1: forced expiratory volume in $1 \mathrm{~s} ; \%$ pred: \% predicted; PD: nasal potential difference. 


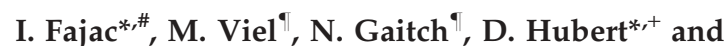

T. Bienvenu ${ }^{\top, \S, f}$

*Université Paris Descartes, "Service d'Explorations Fonctionnelles, Assistance Publique - Hôpitaux de Paris, Hôpital Cochin, "Laboratoire de Biochimie et Génétique Moléculaires, Hôpital Cochin, 'Service de Pneumologie, Assistance Publique Hôpitaux de Paris, Hôpital Cochin, ${ }^{\S}$ Université Paris Descartes, Institut Cochin, CNRS (UMR 8104), and ${ }^{f}$ Inserm U567, Paris, France.

Correspondence: T. Bienvenu, Laboratoire de Biochimie et Génétique Moléculaires, Hôpital Cochin, 123 boulevard de Port Royal, 75014, Paris, France. E-mail: thierry.bienvenu@ inserm.fr

Statement of Interest: None declared.

\section{REFERENCES}

1 Fajac I, Viel M, Sublemontier S, et al. Could a defective epithelial sodium channel lead to bronchiectasis. Respir Res 2008; 9: 46-53.
2 Azad AK, Rauh R, Vermeulen F, et al. Mutations in the amiloridesensitive epithelial sodium channel in patients with cystic fibrosislike disease. Hum Mutat 2009; 30: 1093-1103.

3 Samaha FF, Rubenstein RC, Yan W, et al. Functional polymorphism in the carboxyl terminus of the $\alpha$-subunit of the human epithelial sodium channel. J Biol Chem 2004; 279: 23900-23907.

4 Tong Q, Menon AG, Stockand JD. Functional polymorphisms in the $\alpha$-subunit of the human epithelial $\mathrm{Na}^{+}$channel increase activity. Am J Physiol Renal Physiol 2006; 290: F821-F827.

5 Sugiyama T, Kato N, Yamori Y, et al. Evaluation of selected polymorphisms of the mendelian hypertensive disease genes in the Japanese population. Hypertens Res 2001; 24: 515-521.

6 Des Georges M, Guittard C, Altieri JP, et al. High heterogeneity of CFTR mutations and unexpected low incidence of cystic fibrosis in the Mediterranean France. J Cyst Fibros 2004; 3: 265-272.

7 Chillon M, Casals T, Mercier B, et al. Mutations in the cystic fibrosis gene in patients with congenital absence of the vas deferens. $N$ Engl J Med 1995; 332: 1475-1480.

8 Mutesa L, Azad AK, Verhaeghe C, et al. Genetic analysis of Rwandan patients with cystic fibrosis-like symptoms: identification of novel cystic fibrosis transmembrane conductance regulator and epithelial sodium channel gene variants. Chest 2009; 135: 1233-1242.

DOI: $10.1183 / 09031936.00057309$

\section{Diagnosis of a posterior mediastinal goitre via endobronchial ultrasound-guided transbronchial needle aspiration}

\section{To the Editors:}

Endobronchial ultrasound-guided transbronchial needle aspiration (EBUS-TBNA) is a rapidly developing diagnostic tool. Its utility as a minimally invasive and safe procedure in mediastinal node staging in lung cancer is defined and gaining widespread acceptance. The role of this procedure in the diagnosis of benign diseases is less clear. We report the first case of a posterior mediastinal intrathoracic goitre diagnosed with the aid of EBUS-TBNA.

A 46-yr-old female was referred to the outpatient chest clinic for further investigation of a superior mediastinal mass. She complained of a 4-month history of progressive shortness of breath on exertion. She denied any dysphagia. Examination was unremarkable. A chest radiograph showed a right superior mediastinal mass. Her thyroid function tests were within normal limits. Contrast-enhanced thoracic computed tomography revealed a large posterior mediastinal mass, containing areas of punctate calcification (fig. 1). There was no associated mediastinal adenopathy and the lung parenchyma was normal. Histological confirmation was considered necessary to the planning of surgical intervention. Oesophageal endoscopic ultrasound-guided needle aspiration was abandoned, as the scope was unable to pass down the oesophagus due to extrinsic compression. We performed endobronchial ultrasound guided transtracheal needle aspiration of the posterior mediastinal mass under conscious sedation, using a linear array ultrasonic bronchoscope and a 22-gauge needle. A large heterogeneous mass was identified adjacent to the posterior wall of the upper trachea. Three separate passes into the mass were performed. The procedure was well tolerated with no complications. Cytology revealed low cuboidal and columnar epithelium with follicle formation containing small amounts of colloid. Thyroglobulin (fig. 2) and thyroid transcription factor 1 stains were positive, confirming thyroid origin. A diagnosis of posterior mediastinal intrathoracic goitre was made. The patient was referred for total thyroidectomy, which was successfully performed using a transcervical approach. The histology was in keeping with a benign multinodular goitre with areas of cystic change, old and recent haemorrhage, fibrosis and patchy calcification. No evidence of malignancy was found.

The differential diagnosis of a posterior mediastinal mass is wide and includes neurogenic tumours, Castleman disease, bronchogenic cysts, Bochdalek hernia, mesenchymal tumours and intrathoracic goitre. The radiological features of a goitre include: heterogeneous attenuation, focal punctate or curvilinear calcification within the mass, rapid and prolonged enhancement of the mass after contrast injection, and continuity of the mass with one of the lobes of the thyroid gland [1].

The majority of intrathoracic goitres are acquired and arise from the cervical thyroid gland. In 1934, LAHEY and SWINTON [2] proposed anatomic factors that facilitate cervical goitres to extend downward into the mediastinum through the thoracic inlet as 